\title{
Staphylococcus equorum
}

National Cancer Institute

\section{Source}

National Cancer Institute. Staphylococcus equorum. NCI Thesaurus. Code C86758.

A species of facultatively anaerobic, Gram positive, cocci shaped bacteria in the phylum Firmicutes. This species is positive for catalase, urease and alkaline phosphatase and negative for coagulase and oxidase. It can ferment mannose, fructose, and arabinose but not cellobiose, fucose or xylitol. S, equorum is a part of the normal flora of human skin, involved in sausage and cheese production and is not known to be a pathogen. 